\title{
Speech in Noise Ability in Patients with Central Auditory Processing Disorders (CAPDs)
}

\section{Original Article}

\author{
Wessam Mostafa Essawy, Nihal M. Medhat Abd El-Ghaffar, Mona Ahmed Kotait
}

Department of Otolaryngology, Faculty of Medicine, Tanta University, Gharbia, Egypt.

\begin{abstract}
Background: Central auditory processing disorders (CAPDs) tend to affect an individual's ability to understand speech, especially in difficult listening conditions.

Objective: To study speech-in-noise (SIN) ability in cases of central auditory processing disorders (CAPDs) subjectively by Arabic Hearing in Noise Test (A-HINT) and objectively by Speech-evoked auditory brainstem response (S-ABR) with ipsilateral noise.

Patients and Methods: Subjects included in this study will be divided into 2 groups: a control group which included 30 normal hearing subjects (17 males and 13 females). Their age ranged from 6 to 17 years. All subjects had normal developmental milestones and normal speech development. The Study group included 30 normal hearing subjects (18 males and 12 females). Their age ranged from 7 to 16 years. These subjects had central auditory processing disorders (CAPDs) diagnosed by central auditory screening tests. Both groups were tested using Arabic HINT and Speech-evoked auditory brainstem response (S-ABR) with ipsilateral noise.

Results: Comparison between control and study groups as regards the Arabic HINT revealed statistically significant differences in noise conditions. Using S-ABR significant difference was found in onset and offset response waves latency in noise conditions also.

Conclusion: Patients with CAPDs have speech in noise ability affection proved by affected HINT in noise conditions and by delayed S- ABR with ipsilateral noise latencies in onset- offset responses.
\end{abstract}

Key Words: Arabic hearing in noise test (A- HINT), central auditory processing disorders (CAPDs), speech in noise (SIN), speech-evoked auditory brainstem response (S-ABR).

Received: 14 April 2021, Accepted: 20 May 2021

Corresponding Author: Wessam Mostafa Essawy, Department of Otolaryngology, Faculty of Medicine, Tanta University, Gharbia, Egypt, Tel.: 01064868286, E-mail: wessamessawy@yahoo.com

ISSN: 2090-0740, 2021

\section{INTRODUCTION}

Many factors affect speech in noise (SIN) ability such as the characteristics of the speech signal,signalto- noise ratio (SNR) and the listener's degree of hearing impairment. Moreover, speech innoise (SIN) ability can be considered the main functional communication ability that can't be evaluated by using the hearing routine evaluation tests $^{[1]}$.

Speech-in-noise (SIN) perception needs particular demands in older adults and children withdifficulties in auditory processing (e.g., dyslexia, auditory processing disorders, specific language impairment, autism spectrum disorder, and attention deficit hyperactivity disorder). Even with the absence of peripheral hearing loss, these speech perception difficulties may be arising from dysfunction in more central levels in the auditory system, including subcortical structures ${ }^{[2]}$.

Speech-in-noise (SIN) is a highly complex task affected by reciprocal sensory-cognitiveinteractionsin the brain ${ }^{[3]}$.

Hearing- in-Noise Test (HINT) was developed by (Nilsson et al. 1994) in English language. Itprovides an efficient and reliable method for evaluating the individual's supra-threshold speech understanding ability in quiet and noise using adaptive testing procedures[4].

ArabicHINT(A-HINT) was developed by (Essawy et al., 2019). This test can provide a reliable and efficient tool to estimate hearing handicap, SIN ability, directional hearing, and hearing aid benefits and to perform comparison between hearing aids ${ }^{[5]}$.

Auditory brainstem responses to speech (S-ABR) can be used objectively to evaluate successful SIN perception. Understanding neural processing at the brainstem level may assist in understanding the outcomes in varied populations such as individuals with hearing loss, language disorders and learning deficits ${ }^{[6]}$. Although several complex stimuli have been used to elicit the ABR, speech (specifically/da/) has most commonly been used. A speech 
stimulus is particularly useful, as it can provide cues to how temporal and spectral features are preserved in the brainstem. When elicited with the stimulus /da/, the subcortical response emerges as a wave form of seven identifiable peaks, labeled V, A, C, D, E, F, and O. This response is known as the speech-evoked ABR. Waves $\mathrm{V}$ and $\mathrm{A}$ reflect the onset of the response, wave $\mathrm{C}$ the transition region, waves $\mathrm{D}, \mathrm{E}$ and $\mathrm{F}$ the periodic region (i.e., the frequency following response) and wave Otheoffset of the response ${ }^{[7]}$.

Speech-evoked ABR has also been studied with stimuli presented in noise. Laboratory studies confirm that S-ABR recording in noise increases sensitivity to auditory processing and relateddisorders. Speech-evoked ABR may be used as a tool to objectively measure and quantify theeffects of noiseand mayalso shed light on whysome people have more difficulty in noise than others ${ }^{[8]}$.

The purpose of this work is to study speech-in-noise ability in cases of central auditory processing disorders (CAPDs) subjectively by Arabic Hearing in Noise Test (A-HINT) and objectively by speech ABR with ipsilateral noise.

\section{PATIENTS AND METHODS}

This cross-sectional study was carried out at the Audiovestibular Medicine Unit, Tanta University, Egypt from February 2019 to January 2020.

The idea of the research was explained in detail to the participants. Subjects who agreed toparticipate signed an informed consent. The participation is voluntary; participant may discontinue participation at any time without penalty or loss of benefits. Every participant had acode number. Results of this research are used only in scientific purpose. The duration of thestudy ranged from 6-12 months. The protocol of the study was approved by the ethical committee(32533/07/18).

Subjects included in this study were divided into 2 groups: A control group whichconsisted of 30 normal hearing subjects with normal developmental milestones and normal speech development. Their age ranged from 6 to 17 years. Normal hearing sensitivity is definedas having pure-tone air-conduction thresholds less than or equal to $25 \mathrm{~dB} \mathrm{HL}$ at audiometric test frequencies $250 \mathrm{~Hz}$ to $8000 \mathrm{~Hz}$. Both the pure tone and speech audiometry tests were conducted using a GSI-61 audiometer (GrasonStadler, Eden Prairie, Minnesota, USA). All participating subjects had normal middle ear function as determined by type (A) tympanogram, with present Ipsilateral and contralateral acoustic reflex thresholds in both ears by using Interacoustics AT235 tympanometry. Central Screening auditory tests (LPF, Memory tests, DDT and CST) were applied to all control group subjects revealing normal test results to exclude the presence of any central processing abnormalities in the control group. The study group consisted of 30 subjects with central auditory processing disorders (CAPDs) diagnosed by central auditory screening tests. Screening Central auditory tests, used for diagnosing the study group, included Low Pass Filter for children (LPF), Memory Tests (Recognition memory, Memory for content and Memory for sequence) and Dichotic speechtests including Dichotic Digits Test (DDT) and Competing Sentences Test (CST)). Their ages ranged from 7 to 16 years. Any subjects with any hearing complaints, history of unilateral orbilateral otological diseases or family history of hearing loss, general health problems (e.g. any endocrinal, vascular, renal, cardiovascular, or neurological complaints) were excluded from this study.

All participates in this study were subjected to Arabic Hearing in Noise Test (A-HINT) in Quietand Noise front $\left(0^{\circ}\right)$ by using AC 40 clinical audiometer, loudspeakers, CD player: Thomson Cs 96 and CD of pre-recorded calibrated test material of HINT sentences list in Arabic Language mixed with speech noise. The test required a sound-treated room with two loudspeakers, a chair, a compact disk player and an audiometer. The two loudspeakers were positioned, so that the center of the subject's headisonemeter from each loudspeaker. The loudspeakers are separated by a $90^{\circ}$ azimuth at the ear level of the tested subject. Sentences speech recognition threshold (sSRT)was measured in quiet and in noise. Sentences speech material location remained fixed at $0^{\circ}$ in all tested conditions. The location of noise source differed in three tested conditions: Noise front $\left(0^{\circ}\right)$, Noise $\left(90^{\circ}\right)$ and Noise $\left(270^{\circ}\right)$. In standard HINT test the three noise conditions (Noise $\left(0^{\circ}\right),\left(90^{\circ}\right)$ and $\left(270^{\circ}\right)$ can be done by changing noise source but in the current study, we only used Noise $0^{\circ}$ condition to be compared easily correlated with the results of Speech ABR in noise.

The sentence lists were administered using adaptive testing procedure according to HINT guidelines (House Ear Institute, 1995). In quiet condition, the starting level was $30 \mathrm{~dB}$ SL (referred to SRT by loudspeaker). In noise condition, the noise levelwas fixed at $65 \mathrm{~dB}(\mathrm{~A})$, whereas the intensity levels of sentences were adjusted according to theparticipant's response. The sentence was initially presented at $-5 \mathrm{~dB}$ signal-to-noise (SNR) and the sentence presentation level was increased in $4-\mathrm{dB}$ or lowered by $4 \mathrm{~dB}$ according to the patient's response. Thereafter, the adaptive procedure was preceded to the 10th sentence that would have been presented using 2-dB steps. The averaged SNR from the 5th to 10th sentences in a sentence list was regarded as Sentences speech recognition threshold (sSRT) for that list.

Participants were instructed to listen carefully and repeat aloud whatever they heard as much ofthe sentence as possible. The sentences were presented one at a time. The listener is encouragedto guess if they were not sure what was spoken. Speech evoked Auditory Brainstem Response (S-ABR) was conducted for both the study groups using smart evoked intelligent system. The Speechstimuli used was CV syllables $/ \mathrm{da} /$ of $40.05 \mathrm{~ms}$ duration. It was 
presented in quiet at $80 \mathrm{dBSPL}$ and with ipsilateral noise at +10 and +5 SNRs. White noise was presented ipsilaterally at $70 \mathrm{dBSPL}$ and $75 \mathrm{dBSPL}$ using Smart EPs of Intelligent hearing system (IHS).

Stimuli were presented monaurally via electromagnetically shielded insert ear phone. Repetition rate (R.R) was 10.9 per second with alternating polarity. Electrode montage: one high frontal $\mathrm{Fz}$ (positive electrode), one low frontal Fpz (ground electrode). The last two electrodes were placed on the left and the right mastoids (as negative electrode or reference electrode) depending on the recording side. Sweeps number: 1024 sweeps. The analysis epoch (time window): the recording window was (0 to 60) msec. The response was identified by the presence of seven waves $(\mathrm{V}, \mathrm{A}, \mathrm{C}, \mathrm{D}, \mathrm{E}, \mathrm{F}, \mathrm{O})$ using nomenclature previously established for the S-ABR then detecting the absolute latency and amplitude of each wave $\mathrm{e}^{[8]}$.

Fast Fourier transforms (FFTs) were run on brainstem responses to the $[\mathrm{da}]$ in quiet and noise. This was done to measure pitch encoding (i.e., spectral amplitude corresponding to the fundamental frequency F0). Prior to the Fourier transform, zero-padding was applied to increase the resolution of the spectral display. In each case, spectral amplitude was calculated over 40 Hzbins width at $\mathrm{F} 0$ (around $100 \mathrm{~Hz})$. The cross-phaseogram was done to determine the effect of noise on phase. Phase shifts over the response spectrum $(70-1000 \mathrm{~Hz})$ were calculated for the transition $(11-20 \mathrm{msec})$ and steady-state portions $(20-42 \mathrm{msec})$ of the response using MATLAB7. Crossphaseogram is a three dimensional representation of phase differences with the $\mathrm{x}$-axis representing time, the $\mathrm{y}$-axis representing frequency and the third dimension plotted using a color continuum reflecting phase differences between the pair of signals being compared. A phase shift of zero radians indicates no effect of noise on the timing of the brainstem response, this is plotted in green. A positive phase shift indicates that the response in noise is delayed related to the response inquiet, this is plotted in warm colors, with red indicating the greatest difference. Furthermore, a negative phase shift indicates that the response in noise is earlier than the response in quiet, this is plotted in cool colors, with dark blue indicating the greatest difference.

\section{Statistical analysis}

All data were analyzed by SPSS version 22. Continuous data were tested for normality by Shapiro Wilk test. Normally distributed continuous data were expressed as means of \pm standard deviation and Independent Samples Student's T test was used for comparison. While, not normally distributed data were expressed as median and interquartile range (expressed as 25th-75th percentiles), and Mann-WhitneyU test was applied for comparison. $P<0.05$ was considered statistically significant. For correlation, Spearman correlation coefficient was applied to correlate the results of HINT and S-ABR waves latencies and amplitude. rs: correlation coefficient was considered significant at $P<0.05$. In addition, Receiver operating characteristic (ROC) curves were constructed to assess discriminating power (area under the curve), sensitivity, specificity, and accuracy of the studied parameters in predicting the need for predicting the ability of speech in noise by HINT noise, latency of wave O. $P<0.05$ was considered statistically significant.

\section{RESULTS}

Sixty Subjects participated in this study and were divided into 2 groups. A control group which consisted of 30 normal hearing subjects with normal developmental milestones and normal speech development. They were 17 males and 13 females. Their age ranged from (6 to 17) years with the mean of $10 \pm 1.15$ years. The study group consisted of 30 subjects with central auditory processing disorders (CAPDs) diagnosed by central auditory screening tests. They were 18 males and 12 females. Their ages ranged from ( 7 to 16 ) years with the mean of $11 \pm 0.55$ years. A-HINT were recorded for both groups.

All participants were given one practice list each in quiet and noise at $0^{\circ}$ azimuth conditions to befamiliarized with the task. Then for each condition (quiet, noise $0^{\circ}$ ) three lists were given (each listening condition containing three threshold measurements). So, (sSRT) is the mean of the three measurements in both conditions quiet and noise. The mean and range of sSRT in quiet condition was calculated. It was $19.0 \mathrm{~dB}$ (A) ranged from 19.0 to 19 . $0 \mathrm{~dB}(\mathrm{~A})$ in the control group. In the study group, it was $19.0 \mathrm{~dB}(\mathrm{~A})$ ranged from 19.0 to $19.70 \mathrm{~dB}(\mathrm{~A})$. The mean of $\mathrm{S} / \mathrm{N}$ ratio at threshold in the noise $0^{\circ}$ condition across all subjects was-11.0 ranging from -10.15 to -11.0 in the control group. As regards study group, mean of $\mathrm{S} / \mathrm{N}$ ratio at threshold in the noise $0^{\circ}$ condition was -8.65 ranging from -8.0 to -9.70 . On comparing results between the two groups, no statistically significant differences were found in quiet condition (0.090) but a strong statistically significant difference in Noise $0^{\circ}$ condition $\left(<0.001^{*}\right)$ (Table 1$)$.

Table 1: Control and study group HINT results in Quiet and noise 0 showing Median (IQR)and P value.

\begin{tabular}{lccc}
\hline Median (IQR) & Study group & Control group & $P$ value \\
\hline HINT Quiet & $19.0(19.0-19.70)$ & $19.0(19.0-19.0)$ & 0.090 \\
HINT Noise 0 & $-8.65(-8.0$ to -9.70$)$ & $-11.0(-10.15$ to -11.0$)$ & $<0.001 *$ \\
\hline
\end{tabular}

*significant at $P<0.05 \mathrm{IQR}$ : interquartile range. 
S-ABR as well was recorded for both study groups. All waves of S-ABR were $100 \%$ detected in all subjects in the control group and study group in quiet and noise conditions except wave C. Wave $\mathrm{C}$ was detected in 7 subjects in control group and 4 subjects in the study group. When comparing between control and study groups as regards S-ABR waves latencies, standard student t-test revealed no statistically significant difference in both quiet and noise conditions. Comparison of S-ABR waves latencies, in all recording conditions (quiet, $+10 \mathrm{SNR},+5$ SNR and 0 SNR), between control and study groups was done using ANOVA test. Results revealed statistically significant differences between the two groups response in quiet and noise $(+10,+5 \mathrm{SNR}$ and 0 SNR) in both ears. This was found for latency of the onset waves (VA and O) except in wave $\mathrm{A}$ in Noise 0 and wave $\mathrm{O}$ in Quiet condition (Table 2). On the other hand, this test showed no statistically significantdifferences for latency of waves $(\mathrm{C}, \mathrm{D}, \mathrm{E}$ and $\mathrm{F})$ in all recording conditions between both groups (Table 3). Standard student t-test was used to compare amplitudes in control and study groups.

Table 2: Comparison of S-ABR waves latencies, in all recording conditions (Quiet, +10 SNR, +5 SNR and 0 SNR), in onset waves (VA and O) between control and study groups

\begin{tabular}{ccccc}
\hline S-ABR waves Latencies & Recording conditions & Study group Mean \pm SD & Control group Mean \pm SD & $P$ value \\
\hline \multirow{4}{*}{ V } & Quiet & $6.31 \pm 0.21$ & $6.11 \pm 0.13$ & $0.001^{*}$ \\
& +10 SNR & $6.56 \pm 0.27$ & $6.32 \pm 0.23$ & $0.003^{*}$ \\
& +5 SNR & $6.70 \pm 0.21$ & $6.44 \pm 0.25$ & $0.001^{*}$ \\
0 SNR & $6.78 \pm 0.18$ & $6.55 \pm 0.24$ & $0.001^{*}$ \\
Quiet & $7.86 \pm 0.57$ & $7.31 \pm 0.59$ & $0.003^{*}$ \\
A & $8.22 \pm 0.51$ & $7.67 \pm 0.62$ & $0.002^{*}$ \\
& +5 SNR & $8.41 \pm 0.47$ & $8.02 \pm 0.59$ & $0.019^{*}$ \\
0 SNR & $8.52 \pm 0.50$ & $8.24 \pm 0.69$ & 0.129 \\
Quiet & $47.82 \pm 0.86$ & $47.87 \pm 0.83$ & 0.851 \\
& +10 SNR & $48.91 \pm 1.51$ & $48.08 \pm 0.92$ & $0.034^{*}$ \\
& 0 SNR & $49.47 \pm 1.76$ & $48.26 \pm 0.93$ & $0.008^{*}$ \\
\hline
\end{tabular}

*significant $P<0.05$

Table 3: Comparison of S-ABR waves latencies, in all recording conditions (Quiet, +10 SNR, +5 SNR and 0 SNR), in waves (D, E and F) between control and study groups

\begin{tabular}{ccccc}
\hline S-ABR waves Latencies & Recording conditions & Study group Mean \pm SD & Control group Mean \pm SD & $P$ value \\
\hline \multirow{4}{*}{ D } & Quiet & $21.00 \pm 1.30$ & $21.60 \pm 0.63$ & 0.062 \\
& +10 SNR & $21.11 \pm 1.29$ & $21.75 \pm 0.69$ & 0.050 \\
& +5 SNR & $21.31 \pm 1.38$ & $21.85 \pm 0.76$ & 0.119 \\
0 SNR & $21.46 \pm 1.29$ & $21.92 \pm 0.74$ & 0.158 \\
Quiet & $30.91 \pm 1.03$ & $31.19 \pm 0.88$ & 0.338 \\
E & 10 SNR & $31.03 \pm 1.05$ & $31.34 \pm 0.82$ & 0.283 \\
& +5 SNR & $31.17 \pm 1.10$ & $31.40 \pm 0.91$ & 0.462 \\
0 SNR & $31.25 \pm 1.08$ & $31.56 \pm 0.87$ & 0.299 \\
F & Quiet & $39.48 \pm 0.44$ & $39.68 \pm 1.06$ & 0.426 \\
& +10 SNR & $39.60 \pm 0.40$ & $39.81 \pm 1.13$ & 0.425 \\
& +5 SNR & $39.73 \pm 0.37$ & $39.87 \pm 1.14$ & 0.608 \\
\hline
\end{tabular}


The results showed no statistically significant difference in all conditions and in all waves except wave A in +5 SNR condition (Tables 4 and 5). As regards Quietnoise (Q-N) Cross Correlation, between control and study groups in different SNR conditions, standard student t-test showed no statistically significant difference between them (Table 6). Fast Fourier Transform (FFT) as well as cross-phaseogram were also calculated. As regards FFT Using Fourier analysis, a complex waveform consisting of many frequency components is decomposed into a set of sine waves. The magnitude of each sine wave corresponds to the amount of energy contained in the complex waveform at that frequency. On analyzing the response in the frequency domain, spectral maxima corresponding to the stimulus F0 and its harmonics are identified, and the phase and amplitude (modulus of the FFT) of the maxima are recorded (Skoe and Kraus, 2010). The amplitudes of F0 in quiet and in different SNR conditions revealed no statistically significant difference between the two study groups (Table 7).

Table 4: Comparison of S-ABR waves amplitudes, in all recording conditions (Quiet, $+10 \mathrm{SNR},+5 \mathrm{SNR}$ and 0 SNR), in onset waves (VA and $\mathrm{O}$ ) between control and study groups

\begin{tabular}{lcccc}
\hline \multirow{2}{*}{ S-ABR waves Amplitudes } & Recording conditions & $\begin{array}{c}\text { Study group } \\
\text { Median (IQR) }\end{array}$ & $\begin{array}{c}\text { Control group } \\
\text { Median (IQR) }\end{array}$ & $P$ value \\
\hline \multirow{4}{*}{ V } & Quiet & $0.25(0.16-0.29)$ & $0.22(0.14-0.28)$ & 0.387 \\
& +10 SNR & $0.17(0.14-0.29)$ & $0.18(0.15-0.29)$ & 0.626 \\
& +5 SNR & $0.17(0.12-0.27)$ & $0.18(0.14-0.27)$ & 0.903 \\
& 0 SNR & $0.13(0.12-0.21)$ & $0.14(0.12-0.25)$ & 0.712 \\
Quiet & $0.24(0.16-0.28)$ & $0.23(0.14-0.32)$ & 0.724 \\
A & +10 SNR & $0.21(0.19-0.25)$ & $0.22(0.19-00.26)$ & 0.798 \\
& +5 SNR & $0.43(0.26-0.50)$ & $0.26(0.17-0.40)$ & $0.046^{*}$ \\
& 0 SNR & $0.24(0.20-0.40)$ & $0.22(0.16-.029)$ & 0.573 \\
Quiet & $0.33(0.16-0.74)$ & $0.27(0.16-0.74)$ & 0.990 \\
& +10 SNR & $0.26(0.16-0.60)$ & $0.26(0.16-0.47)$ & 0.742 \\
& +5 SNR & $0.19(0.13-0.71)$ & $0.21(0.13-0.46)$ & 0.874 \\
\hline
\end{tabular}

*Significant $P<0.05$ IQR: interquartile range.

Table 5: Comparison of S-ABR waves amplitudes, in all recording conditions (Quiet, +10 SNR, +5 SNR and 0 SNR), in waves (D,E and F) between control and study groups

\begin{tabular}{lcccc}
\hline \multirow{2}{*}{ S-ABR waves Amplitudes } & Recording conditions & $\begin{array}{c}\text { Study group } \\
\text { Median } \pm \text { SD }\end{array}$ & $\begin{array}{c}\text { Control group } \\
\text { Median } \pm \text { SD }\end{array}$ & $P$ value \\
\hline \multirow{4}{*}{ D } & Quiet & $0.28(0.24-0.92)$ & $0.27(0.20-0.51)$ & 0.443 \\
& +10 SNR & $0.31(0.22-0.37)$ & $0.30(0.21-0.44)$ & 0.798 \\
& +5 SNR & $0.23(0.15-0.26)$ & $0.2(0.16-0.31)$ & 0.600 \\
& 0 SNR & $0.21(0.14-0.25)$ & $0.20(0.10-0.23)$ & 0.507 \\
Quiet & $0.38(0.23-0.50)$ & $0.41(0.25-0.64)$ & 0.893 \\
E & +10 SNR & $0.37(0.27-0.47)$ & $0.33(0.28-0.47)$ & 0.971 \\
& +5 SNR & $0.28(0.22-0.46)$ & $0.3(0.22-0.45)$ & 0.808 \\
F SNR & $0.26(0.19-0.43)$ & $0.24(0.14-0.41)$ & 0.538 \\
& Quiet & $0.47(0.28-0.70)$ & $0.46(0.29-0.70)$ & 0.865 \\
& +10 SNR & $0.37(0.28-0.64)$ & $0.40(0.28-0.61)$ & 0.971 \\
& +5 SNR & $0.33(0.22-0.54)$ & $0.38(0.22-0.55)$ & 0.817 \\
\hline
\end{tabular}

*Significant $P<0.05$ IQR: interquartile range. 
Table 6: Comparison between control and study groups as regards Quiet-noise (Q-N) correlations in different SNR conditions.

\begin{tabular}{lccc}
\hline Quiet-noise $(\mathrm{Q}-\mathrm{N})$ correlation & Study group $($ Mean $\pm \mathrm{SD})$ & Control group $($ Mean \pm SD) & $P$ value \\
\hline Quiet/+10 & $0.786 \pm 0.093$ & $0.777 \pm 0.099$ & 0.737 \\
Quiet/+5 & $0.732 \pm 0.126$ & $0.719 \pm 0.129$ & 0.752 \\
Quiet/0 & $0.5 \pm 0.1$ & $0.5 \pm 0.1$ & 0.823 \\
\hline
\end{tabular}

Table 7: Comparison between control and study groups as regards Fast Fourier Transform (FFT) in Quiet and different SNR conditions.

\begin{tabular}{lccc}
\hline FFT & Study group Median (IQR) & Control group Median (IQR) & $P$ value \\
\hline Quiet & $0.044(0.026-0.085)$ & 0.044 & $(0.030-0.085)$ \\
SNR +10 & $0.027(0.019-0.070)$ & $0.027(0.021-0.070)$ & 0.689 \\
SNR +5 & $0.019(0.013-0.075)$ & $0.019(0.013-0.070)$ & 0.654 \\
SNR0 & $0.01(0.00-0.04)$ & $0.01(0.01-0.04)$ & 0.906 \\
\hline
\end{tabular}

The results revealed no statistically significant difference as regards sustained and transition regions between control and study groups (Tables 8 and 9). On the other hand, Cross-phaseogram between response in quiet and noise (zero SNR) in NH subject and other with CAPD showed more noise induced phase shift in the transition region in CAPD than normal indication by the average phase shift and much warm color. There was nearly no change in the steady state region in NH or CAPD (Figure1 and 2). Spearman's rank for correlations between HINT and S-ABR waves latencies and amplitudes. Results showed only positive correlation between HINT and wave O latency in noise conditions. Receiver operating characteristics (ROC) curve analysis was used for predicting the ability of speech in noise by HINT noise, latency of wave $\mathrm{O}$ in $\mathrm{SNR}+10$, $\mathrm{SNR}+5$, SNR0. Figure 3. Depending on ROC curve analysis, we get sensitivity $\%$, specificity $\%$, accuracy $\%$, AUC area under the curve, $95 \%$ CI and $P$ value for HINT and latency of wave O in SNR+10, SNR+5, SNR0 (Table 10).

Table 8: Comparison between control and study groups as regards Cross-phaseogram in transition region in Quiet and different SNR conditions.

\begin{tabular}{lccc}
\hline Transition & Study group Median (IQR) & Control group Median (IQR) & $P$ value \\
\hline Quiet+10 & $0.28752(0.06166-0.46954)$ & $0.28752(0.06724-0.46954)$ & 0.759 \\
Quiet+ 5 & $0.53662(0.34063-0.85010)$ & $0.50467(0.44950-0.85010)$ & 0.906 \\
Quiet 0 & $.93874(0.73662-1.35010$ & $0.93874(0.76272-1.35010)$ & 0.832 \\
\hline
\end{tabular}

Table 9: Comparison between control and study groups as regards Cross-phaseogram in transition region in Quiet and different SNR conditions.

\begin{tabular}{lllc}
\hline Sustained & Study group Median (IQR) & Control group Median (IQR) & $P$ value \\
\hline Quiet+10 & $0.14658(0.08699-0.23159)$ & $0.14658(0.12237-0.38415)$ & 0.587 \\
Quiet+ 5 & $0.18755(0.11750-0.48844)$ & $0.18755(0.14815-0.48844)$ & 0.723 \\
Quiet 0 & $0.21750(0.19055-0.59526)$ & $0.24815(0.19055-0.59526)$ & 0.555 \\
\hline
\end{tabular}




\section{Average Phase Shift within ROI: 0.015791}

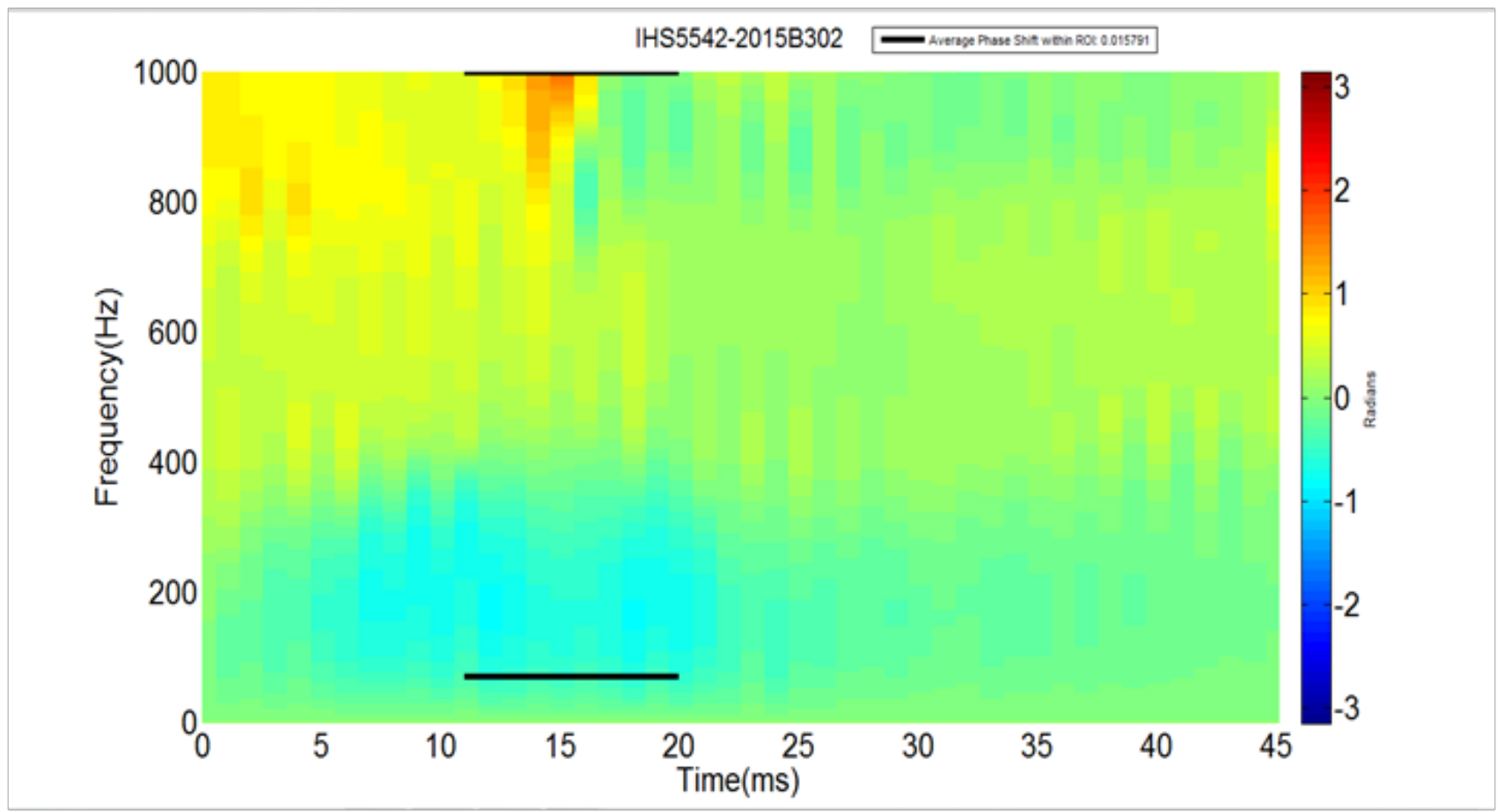

Fig 1: Cross phaseogram from normal hearing subjects $(\mathrm{NH})$

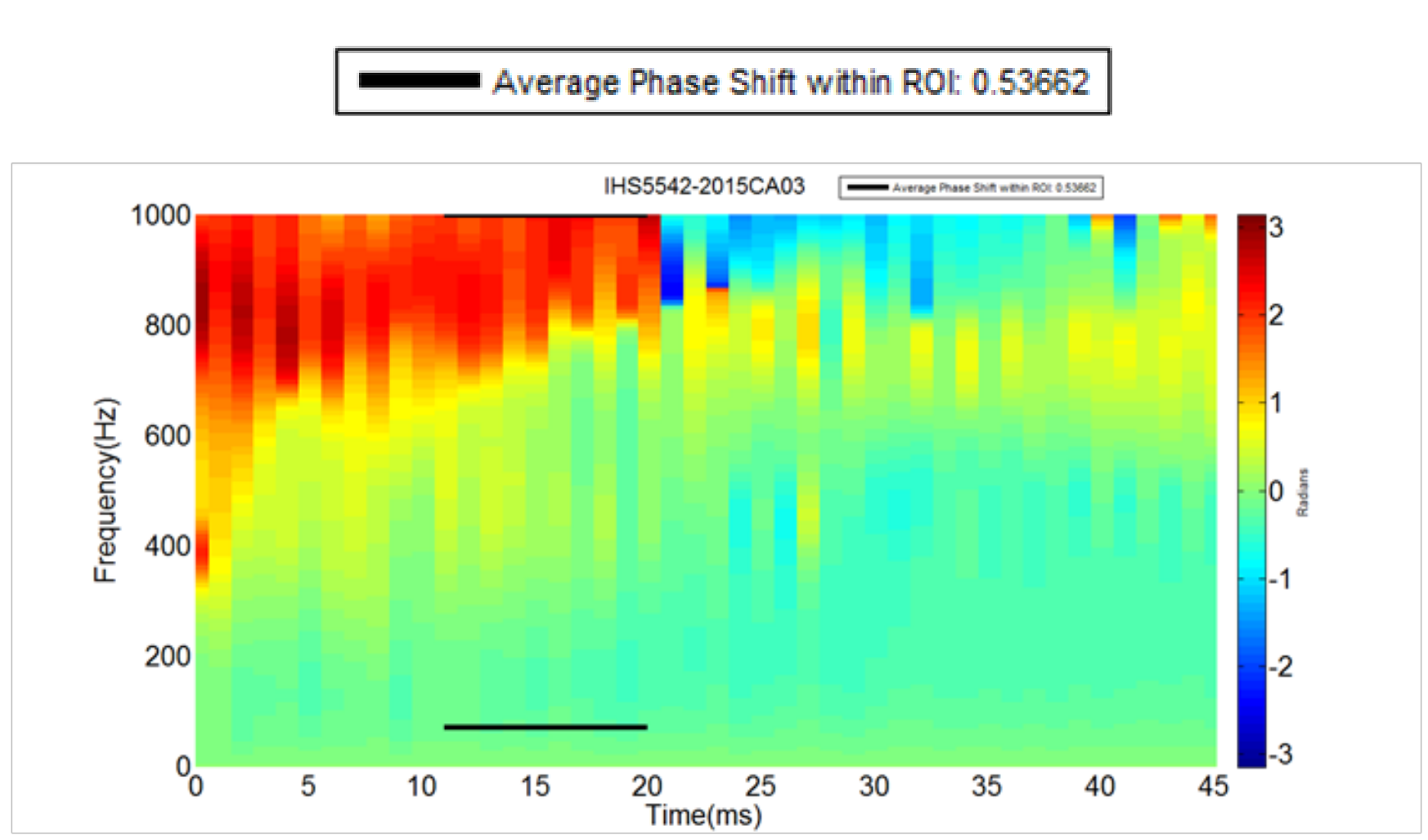

Fig 2: Cross phaseogram from subject with CAPD 


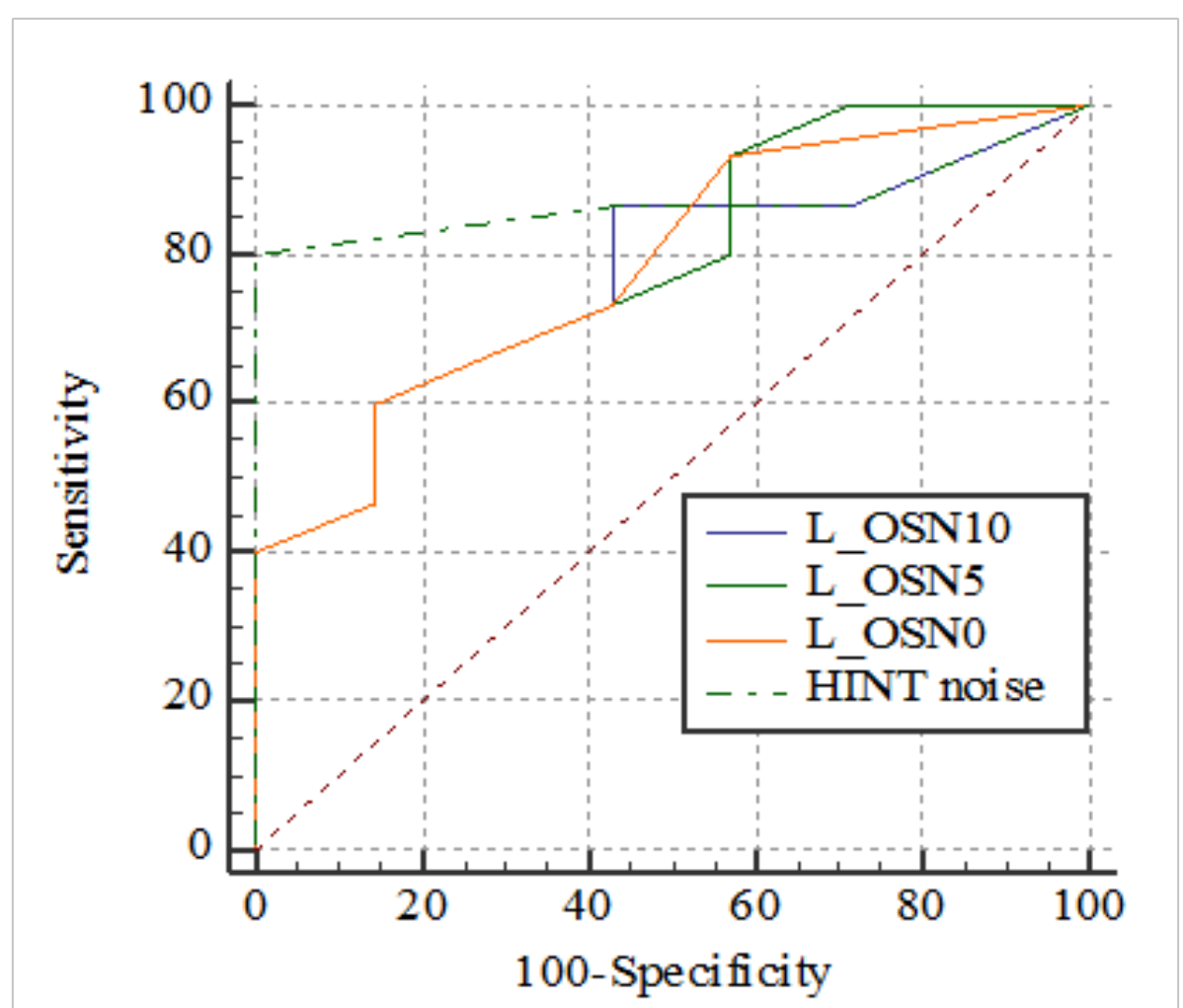

Fig. 3: Receiver operating characteristics (ROC) curve analysis for predicting the ability of speech in noise by HINT noise, latency of wave $\mathrm{O}$ in $\mathrm{SNR}+10, \mathrm{SNR}+5$, SNR0

Table 10: Receiver operating characteristics (ROC) curve analysis HINT noise, latency of wave $\mathrm{O}$ in $\mathrm{SNR}+10$, SNR +5 , SNR0 showing sensitivity $\%$, specificity $\%$, accuracy $\%$, AUC area under the curve, $95 \% \mathrm{CI}$ and P value for HINT and latency of wave O in SNR+10, $\mathrm{SNR}+5, \mathrm{SNR} 0$.

\begin{tabular}{lccccccc}
\hline & Cut off & $\begin{array}{c}\text { Sensitivity } \\
\%\end{array}$ & $\begin{array}{c}\text { Specificity } \\
\%\end{array}$ & Accuracy \% & AUC & 95\%CI & $P$ value \\
\hline HINT noise & $>8.3$ & 80.0 & 100.0 & 77.3 & 0.871 & 0.660 to 0.974 & $<0.001^{*}$ \\
Latency wave OSN10 & $>48.83$ & 60.0 & 85.71 & 68.2 & 0.767 & 0.540 to 0.918 & $0.012^{*}$ \\
Latency wave OSN5 & $>49.25$ & 60.0 & 85.71 & 68.2 & 0.786 & 0.561 to 0.930 & $0.006^{*}$ \\
Latency wave OSN0 & $>49.3$ & 60.0 & 85.71 & 68.2 & 0.786 & 0.561 to 0.930 & $0.005^{*}$
\end{tabular}

Pairwise comparison based on differences between area under the curves revealed no significant differences between other studied parameters $(P>0.05)$

AUC: area under the curve, CI: confidence interval.

* Significant at $P<0.05$. 


\section{DISCUSSION}

Auditory processing disorder (APD) refers to difficulties in the perceptual processing of auditory information in the central nervous system and the neurobiological activity that underlies that processing and gives rise to the electrophysiological auditory potentials. The characteristic symptom of APD involves listening difficulties in the presence of background noise ${ }^{[9]}$. Subjects included in this study were divided into 2 groups. A control group which consisted of 30 normal hearing subjects and a study group which consisted of 30 subjects with central auditory processing disorders (CAPDs) diagnosed by central auditory screening tests. Results of A-HINT showed a strong statistically significant difference in noise $0^{\circ}$ condition. This is a clear subjective indicator of affected speech in noise ability in APD children. Many studies used speech in noise tests in APD patient using SPIN ${ }^{[10]}$ or other tests as $\mathrm{SSW}^{[11]}$ but none used A-HINT. Speech Perception in Noise (SPIN) test allows the exploration of the auditory and language-based functions involved in speech perception in noise, which is not possible with most other speech-in-noise tests. APD is a complex and heterogeneous disorder for which the underlying deficit is currently unclear. SPIN-like tests can potentially be used to identify the nature of the deficits underlying problems with speech perception in noise for this population. A better understanding of the difficulties with speech perception in noise experienced by many listeners with APD should lead to more efficient intervention programs ${ }^{[12]}$. Lagacé et al., 2011 found that children with APD benefit have difficulty in linguistic context when perceiving speech in presence of background noise, in comparison to the control group ${ }^{[10]}$. Several previous studies have shown the utility of speech-evoked ABR in the diagnosis of language based processing deficits like learning disability and specific language impairment; however, missing from literature is a study that has ruled out the existence of comorbidity of such conditions and carefully delineated the efficacy of speech-evoked ABR in children with children with auditory processing disorders and reading deficits. Hence, our study aimed at investigating Speechevoked ABR in children with auditory processing disorders without treading problems.

In this study, comparison of S-ABR waves latencies, in all recording conditions (quiet, $+10 \mathrm{SNR},+5 \mathrm{SNR}$ and 0 SNR), between control and study groups, revealed statistically significant differences found mainly in latency of the onset waves (VA and O). These findings are similar to many studies. (Sanfinsa et al., 2017) used ABR with speech stimuli, abnormalities were significantly observed as prolonged absolute latency values of $\mathrm{V}$ and $\mathrm{A}$ waves, as well as of the VA slope. This suggests a rethinking of how speech sounds are encoded and the functional role of the structures are responsible for generating these waves (the region of the lateral lemniscus and inferior colliculus $)^{[13]}$. Kumar and Singh, 2015 screened 336 school going children in the age range of 8-12 years for presence of central auditory processing deficits. Among the 51 children who were identified as at risk, 15 were randomly selected and served as experimental group. The control group comprised of fifteen age matched children. The inter-group comparison was made using ANOVA, which revealed significant prolongations of latencies of waves $\mathrm{V}$ and $\mathrm{A}$ along with marginal reductions in V/A slope and amplitude of responses to the first formant. Speechevoked ABR are affected in children who are at risk of central auditory processing disorders without reading deficits which probably indicates the presence of abnormal brainstem encoding of speech signal in this population ${ }^{[14]}$. Banai et al., 2007 concludes that as many as $40 \%$ of LDs have abnormal speech-ABRs and that these individuals are also likely to exhibit abnormal cortical processing ${ }^{[15]}$.

The findings of this study suggest the existence of functional impairment in speech processing in the brainstem region, identified by prolonged latencies of $\mathrm{V}$ and $\mathrm{A}$ waves and $\mathrm{VA}$ slope, suggesting that the physiological mechanisms are altered, even without a proven neurobiological abnormality in patients exhibiting scholastic difficulties. Thus, there is a negative impact on the processing of fast acoustic signals in the specialized cortical structures, which do not respond to the sound stimulus in asynchronous and organized fashion and thus render the interpretation and understanding of the meaning difficult for children with scholastic difficulties ${ }^{[14]}$.

On the other hand, Wible et al., 2004 found that the onset of the speech sound /da/, wave V-V(n) of the auditory brainstem response (ABR) had a significantly shallower slope in APD children, suggesting longer duration and/or smaller amplitude. The amplitude of the frequency following response (FFR) was diminished in APD subjects over the 229-686 Hz range, which corresponds to the first formant of the/da/ stimulus, while activity at $114 \mathrm{~Hz}$, representing the fundamental frequency of $/ \mathrm{da} /$, was no difference between groups. Normal indicators of auditory peripheral integrity suggest a central, neural origin of these differences. These data suggest that poor representation of crucial components of speech sounds could contribute to difficulties with higher-level language processes ${ }^{[16]}$.

(Ghannoum et al., 2014) showed statistically significant delaying latencies of waves $\mathrm{V}, \mathrm{A}$ and $\mathrm{F}$ in both ears in APD group compared to their controls. There was a statistically significant diminished amplitude of wave $\mathrm{F}$ in the test group in both ears compared to their controls. There was statistically significant decreased amplitudes of waves D and $E$ in $6-8$ years' subgroup and waves $C$ and $D$ in $8-10$ years'subgroup compared to their controls. In addition, compared to the controls, the amplitude of waves D and E were statistically significantly decreased in the 6-8 years' subgroup, and waves C and D in the 8-10 years' subgroup. They concluded that Speech-ABR response parameters are 
affected in APD children, reflecting abnormalities in brain stem encoding of speech signals ${ }^{[17]}$.

Our explanation of these abnormalities in S-ABR onset waves latencies is delayed central nervous system myelination, particularly of the corpus callosum. This has been suggested to be the underlying dysfunction in several cases of $\mathrm{APD}^{[18]}$. Another hypothesis is abnormal low level of activity of the medial olivocochlear bundle (MOC) system. Muchnik et al. (2004) have found that a high proportion of children with APD present reduced activity of the MOC system, which may indicate an auditory inhibitory dysfunction and affect their ability to perceive speech in the presence of background noise ${ }^{[19]}$. Findings from Cameron and Dillon (2008) support the hypothesis that children with suspected APD have a deficit in spatial stream segregation involving an inability to suppress unwanted signals ${ }^{[20]}$.

\section{CONCLUSION}

Central Auditory Processing Disorders (CAPDs) tend to affect an individual's ability to understand speech, especially in difficult listening conditions. Our study aimed to study speech in noise (SIN) ability in cases of central auditory processing disorders (CAPDs) subjectively by Arabic Hearing in Noise Test (A-HINT) and objectively by Speech-evoked auditory brain stem response (S-ABR) with ipsilateral noise. Patients with CAPDs have speech in noise ability affection proved by affected HINT in noise conditions and by delayed S-ABR with ipsilateral noise latencies in onset-offset responses.

\section{ACKNOWLEDGEMENT}

We are thankful to all members of the Audio-vestibular Unit, (Professors, doctors, nurses and workers) Tanta University. Also, thanks for the cooperative participants of this study who helped us a lot for the service of humanity.

\section{CONFLICT OF INTEREST}

There are no conflicts of interest.

\section{REFERENCES}

1. Parrish D.: Monaural speech-in-noise thresholds for sentences using the hearing in noise test (HINT).MD Thesis and Dissertations. November,2003.Tampa, Florida. Paper1446.

2. Anderson S. and Kraus N.: cABR: A neural probe of speech-in noise processing. In Dau T., Jepsen M., Cristensen-Dalsgaard J. and Poulsen T. (Eds.), Proceedings of ISAAR 2012: Speech perception and auditory disorders. The Danavox Jubilee Foundation, 2012.

3. Anderson S., Parbery-Clark A., White-Schwoch T. and Kraus N.: Auditory brainstem response to complex sounds predicts self-reported speech-innoise performance. Journal of Speech Language and Hearing Research, 2013,56 (1): 31-43.

4. Nilsson M.J; Soli S.D. and Sullivan J.A.: Development of the Hearing in Noise Test for the measurement of speech reception thresholds in quiet and in noise. J. Acoust. Soc. Am, 1994; 95:1085-1099.

5. Wessam W. M; Kolkaila E. A; Kabbash I. A. and Emara A.A.:Development and standardization of new hearing in noise test in Arabic language. Int J Otorhinolaryngol HeadNeck Surg. 2019 Nov;5(6):1501-1506 http:// www.ijorl.com pISSN 2454-5929 | eISSN 2454-5937

6. Anderson S. and Kraus N.: Objective Neural Indices of Speech-in-Noise Perception. Trends in Amplification, 2010;14 (2): 73-83.

7. Skoe E. and Kraus N.: Auditory Brain Stem Response to ComplexSounds: A Tutorial. EarandHearing,2010;31: 301-324.

8. Johnson K., Nicol T. And Kraus N.: Brain Stem Response to Speech: A Biological Marker of Auditory Processing. Ear and Hearing,2005;26 (5): 424-434.

9. American Speech-Language-Hearing Association. 2005. (Central) Auditory Processing Disorders. [technical report]. Available at http://www.asha.org/ members/deskref-journals/deskref/default.

10. Lagacé J. E, Jutras T. B, Gigu C. and Gagné J. P.: Speech perception in noise: Exploring the effect of linguistic context in children with and without auditory processing disorder. International Journal of Audiology 2011; 50: 385-395.

11. Wiemes G. R. M, Kozlowski L, Mocellin M, Hamerschmidt R. and Schuch L. H.: Cognitive evoked potentials and central auditory processing in children with reading and writing disorders. Brazilian Journal of Otorhinolaryngol. 2012;78(3):91-97.

12. Lagacé J. E, JutrasT. B. and Gagné J. P.: Auditory processing disorder and speech perception problems in noise: finding the underlying origin. Am J.Audiol. 2010 Jun;19(1):17-25.doi: 10.1044/10590889(2010/09-0022). PMID: 20308289 DOI: 10.1044/1059-0889(2010/09-0022)

13. Sanfins M, Borges L, Francisca $M$ and Santos C: Speech auditory brain response (SpeechABR) in the differential diagnosis of scholastic under achievement. 
14. Kumar P. and Singh N. K. : BioMARK as electrophysiological tool for assessing children at risk for (central) auditory processing disorders without reading deficits. Hear Res. 2015 Jun;324:54-8.doi: 10.1016/j.heares.2015.03.001.

15. Banai K, Abrams D. and Kraus N. : Sensory-based learning disability: Insights from brainstem processing of speech sounds. Int J Audiol. 2007 Sep;46(9):524532. doi:10.1080/14992020701383035.

16. Wible B, Nicol T. and Kraus N.: Atypical brainstem representation of onset and formant structure of speech sounds in children with language-based learning problems. BiolPsychol.2004Nov; 67(3): 299-317.doi: 10.1016/j.biopsycho.2004.02.002.

17. Ghannoum M. T, Shalaby A. A, Dabbous A. O, AbdEl-Raouf E. R. and Abd-El-Hady H.S. : Speech evoked auditory brainstem response in learning disabled children. Hearing, Balance and Communication, 2014; 12 (3): 31-35

18. Liasis A, Bamiou D-E, Campbell P, Sirimanna T. and Boyd S.: Auditory event-related potentials in the assessment of auditory processing disorders: A pilot study.Neuropediatrics, 2003;34:23 -29.

19. Muchnik C, Roth D. A-E, Othman-Jebara R, Putter-Katz H. and Shabtai E.L.: Reduced medial olivocochlear bundle system function inchildren with auditory processing disorders. Audiol Neurootol, 2004; 9:107- 114 .

20. Cameron S. and Dillon H.: The listening in spatialized noise - sentences test (LISN-S): Comparison to the prototype LISN and results from children with either a suspected (central) auditory processing disorder or a confirmed language disorder. JAm AcadAudiol, 2008; 19: 377-391. 